\title{
A Modified Lotka-Volterra Model for Diffusion and Substitution of Multigeneration DRAM Processing Technologies
}

\author{
Hui-Chih Hung, Yu-Chih Chiu, and Muh-Cherng Wu \\ Department of Industrial Engineering and Management, National Chiao Tung University, 1001 University Rd., Hsinchu 30010, Taiwan \\ Correspondence should be addressed to Hui-Chih Hung; hhc@nctu.edu.tw
}

Received 10 December 2016; Accepted 2 May 2017; Published 21 May 2017

Academic Editor: Fazal M. Mahomed

Copyright (C) 2017 Hui-Chih Hung et al. This is an open access article distributed under the Creative Commons Attribution License, which permits unrestricted use, distribution, and reproduction in any medium, provided the original work is properly cited.

\begin{abstract}
We attempt to develop an effective forecasting model for the diffusion and substitution of multigeneration Dynamic Random Access Memory (DRAM) processing technologies. We consider market share data and propose a modified Lotka-Volterra model, in which an additional constraint on the summation of market share is introduced. The mean absolute error is used to measure the accuracy of our market share predictions. Market share data in DRAM industries from quarter one (Q1) of 2005 to 2013 Q4 is collected to validate the prediction accuracy. Our model significantly outperforms other benchmark forecasting models of both revenue and market share data, including the Bass and Lotka-Volterra models. Compared to prior studies on forecasting the diffusion and substitution of multigeneration technologies, our model has two new perspectives: (1) allowing undetermined number of multigeneration technologies and inconsecutive adoption of new technologies and (2) requiring less data for forecasting newborn technologies.
\end{abstract}

\section{Introduction}

Dynamic Random Access Memory (DRAM), a semiconductor product that stores data bits in the form of capacitors and transistors, is a necessary and critical component widely used in information technology (IT) products. Growing with the Personal Computer (PC) boom, DRAM sector is one of the lead products in the semiconductor industry for last decades [1]. In today's modern IT industry, the demand of DRAM is also driven by the data storage and big data retrieval in social media and cloud computing [2]. Beyond that, mobile DRAM also drove record-high profits for DRAM vendors during the second half of 2014 [3]. The total revenue of DRAM products reached 39 billion US dollars in 2010, which accounted for $12.8 \%$ of the revenue of the semiconductor industry. According to Taiwan's Industrial Technology Research Institute (ITRI), almost 70\% of DRAM sales are in PCs (including notebooks) and servers. About $10 \%$ of DRAM sales are in computer peripheral products and $20 \%$ are in mobile communications and consumer electronics products. Therefore, the prices of DRAM products have an important influence on the costs of other industries, including computers, mobile communications, and consumer electronics industries.

Because of price competition, global DRAM manufacturers continue to upgrade their technologies and production processes. The development of new technologies allows smaller chips, which enables wafers to be cut into more chips. This greatly reduces the production cost per chip and enhances price competition. The market shares of different DRAM processing technologies are shown in Figure 1 [4-6]. In quarter one (Q1) of 2005, processing technology based on 100-120 nanometers $(\mathrm{nm})$ constituted mainstream DRAM manufacturing, and its market share reached $85 \%$. Then, processing technology based on 90 nanometers began to develop. By 2010 Q4, processing technology based on 40 nanometers became mainstream, and its market share reached 55\%. Currently, processing technology based on 30 nanometers is developing and has a market share of $21 \%$. Figure 1 shows that the rapid substitution of processing technology is common in the DRAM industry. 


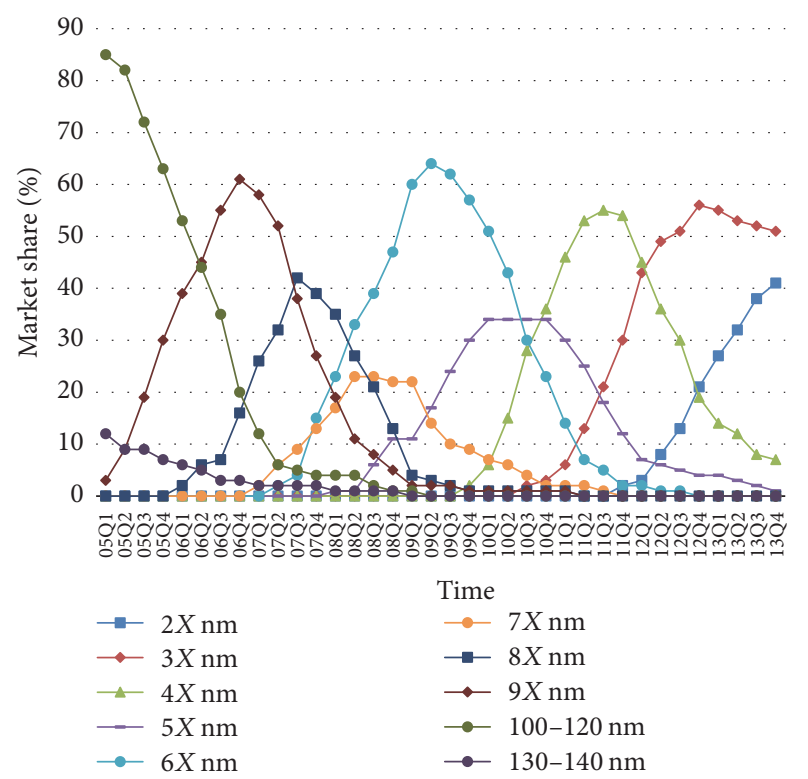

FIGURE 1: Rapid substitution of DRAM processing technologies. Data sources: DRAMeXchange, IHS iSuppli, and Morgan Stanley Research (2014). Data collation: Industrial Technology Research Institute of Taiwan and Taiwan Institute of Economic Research.

The rapid substitution of processing technologies makes prediction important in the DRAM industry. Accurate prediction of the substitution of processing technology helps DRAM manufacturers to invest appropriately in research and development and get the right timing for updating manufacturing equipment. With accurate prediction, technology leaders can plan and allocate resources for the next generation of technologies and maintain their leading positions. Technology followers can ignore obsolete technology upgrades to reduce their capital needs and operational risks. Hence, producers need good forecasting models for predicting the substitution of DRAM processing technology.

Many models, such as the Bass model and the LotkaVolterra model, are widely used to forecast the diffusion and substitution of two technologies. The Bass model mimics the diffusion process of an innovative product or technology and shows how that new product or technology can be adopted as an interaction between users and potential users. The Lotka-Volterra model is a mathematical model that mimics the dynamics of two species competing for common resources. Both models are built based on the data of adoption/shipment amounts and/or product revenues, which may be affected by industry long-term trends, economic cycles, and other confounding factors. Given this sort of interference, both models may yield inaccurate predictions.

For multiple competing technologies, Peterka extended the empirical model of Fisher and Pry to forecast the competition of multiple technologies based on market share data [7, 8]. Norton and Bass generalized the Bass model to encompass the diffusion and substitution of successive generations of DRAM processing technologies [9]. Though establishing significant milestones, these forecasting models for multigeneration of technologies have the following three issues.
First, the number of multigeneration technologies must be preset. Second, inconsecutive adoption of new technologies is not allowed. Third, the number of fitting parameters grows quickly with the number of multigeneration technologies and more data is required for prediction accuracy. However, the total number of multigeneration technologies may not always be predictable in practice. We also observe that new generations of DRAM processing technologies may come to premature end without adoption.

Our study concentrates on the above three issues. We first consider dividing lines to partition the relatively old and new DRAM processing technologies and their market share data is adopted. Then, a modified Lotka-Volterra model with an additional constraint on the summation of market shares is proposed.

To measure the prediction accuracy of market share, we use the mean absolute error (MAE). Market share data in DRAM industry from 2005 Q1 to 2013 Q4 are collected to validate the prediction accuracy. Our modified Lotka-Volterra model significantly outperforms other benchmark forecasting models, which include the Bass and Lotka-Volterra models based on either revenue or market share data. Also, our modified Lotka-Volterra model may help DRAM mangers determine the timing of capital investment decisions. More importantly, this work sheds light on how to appropriately apply the market share data in forecasting technological diffusion and substitution.

The remainder of this paper is organized as follows. Related literature is reviewed in Section 2 and our modified Lotka-Volterra model is proposed in Section 3. In Section 4, we present our data on the DRAM industry and empirical studies are implemented to verify the performance of our model. In Section 5, we summarize our work and discuss directions for future research.

\section{Literature Review}

In this section, we review the Bass and Lotka-Volterra models and other variations.

2.1. Bass Model. The Bass model was introduced by Bass in 1969 [10]. The model describes the diffusion process of new products or technologies being adopted and the interaction between users and potential users. Bass relied on diffusion theory to model the timing of adoption that leads to a particular S-shaped growth pattern for new products or technologies [11]. The diffusion rate of a new product in the market is described by the following differential equation:

$$
\frac{d N(t)}{d t}=(p+q N(t))(M-N(t))
$$

where $N(t)$ is the cumulative number of adopters or users at time $t$ and $M$ is the potential market size. The parameter $p$ is the coefficient of innovation, and $q$ is the coefficient of imitation.

The Bass model and variations have been widely used for diffusion and sales forecasting models of new products or technologies. For example, Park et al. used the Bass 
model to develop a market penetration forecasting model of Hydrogen Fuel Cell Vehicles incorporating infrastructure and cost reduction effects [12]. Cheng used the Bass model to construct predictive models of technology and market diffusion for advanced ceramic powder materials. He examined the relationship over time between technology diffusion and market diffusion [13]. Tsai et al. considered the effect of price factors and modified the Bass model to study the diffusion of the new liquid crystal display TVs [14]. Kong and Bi studied the diffusion of electric vehicles in China with the Bass model. They examined two common business models of battery leasing and vehicle purchasing and analyzed the impact and influence of social network to the innovation coefficient and imitation coefficient [15].

2.2. Lotka-Volterra Model. The Lotka-Volterra model was first proposed by Lotka in 1925 [16]. This model uses two nonlinear differential equations to describe the dynamics and balance of a biological system in which two species interact as predator and prey. Volterra used Lotka's model and his own data to analyze the rise and fall of fish populations in the Adriatic [17]. Lotka and Volterra based their model on the logistic equation, including terms to account for species' interactions. The following two differential equations are used to describe how two species' population growth rates evolve over time:

$$
\begin{aligned}
& \frac{d x(t)}{d t}=\left(a_{1}+b_{1} x(t)+c_{1} y(t)\right) x(t), \\
& \frac{d y(t)}{d t}=\left(a_{2}+b_{2} y(t)+c_{2} x(t)\right) y(t),
\end{aligned}
$$

where $x(t)$ and $y(t)$ represent the populations of two competing species at time $t$, respectively. The terms $x^{2}$ and $y^{2}$ represent internal self-interaction of the same species. The term $x y$ represents the interaction between the two species. The set of differential equations contains basic parameters that affect the growth rates of both species. Parameter $a_{i}$ is the logistic parameter of geometric growth for species $i$ when it is evolving. Parameter $b_{i}$ is the limitation parameter of the niche capacity for species $i$. Parameter $c_{i}$ is the interaction parameter with the other species.

The Lotka-Volterra model has been widely used to investigate relationships between biological species. For example, Smitalova and Sujan proposed a competitive relationship between two competing species [18]. Geijzendorffer et al. used the Lotka-Volterra model to predict the long-term coexistence patterns of grassland species [19].

Outside the field of biology, Goodwin introduced the model into the field of economics by applying it to business cycles [20]. Subsequently, researchers used this model to investigate competitive relationships between different industries, products, and technologies. Kreng and Wang applied the Lotka-Volterra model to analyze the competition between LCD TVs and PDP TVs [21]. Kim et al. considered the dynamic competition of mobile phone subscription in Korea and applied the Lotka-Volterra model to show the commensalism relationship [22]. Watanabe et al. studied the transition process from monochrome TV to color TV in the 1960s and used the Lotka-Volterra model to forecast the transition from analog broadcasting to digital broadcasting in Japan [23].

\subsection{Diffusion and Substitution of DRAM Processing Technolo-} gies. Forecasting market diffusion is important when a new product or technology is under development. The Bass model remains the most adopted and extends diffusion model. Unlike the Bass model, the Lotka-Volterra model incorporates interactions between competitors. Although they differ crucially in their treatment of competitors' interactions, the Bass and Lotka-Volterra models have both been used to forecast the diffusion and substitution of new products, technologies, technologies, and industries.

For example, Tsai and Li found that the development of the integrated circuit (IC) design industry had a positive influence on the IC manufacturing and IC packing industries [24]. They used the Bass and Lotka-Volterra models to verify the commercial relationships between producers in this Taiwanese IC cluster. Chiang explored the innovation growth of $200 \mathrm{~mm}$ and $300 \mathrm{~mm}$ silicon wafers from Taiwan with both the Bass and Lotka-Volterra models. They found that the forecasting performances of the both models are similar [25]. Chiang and Wong used the two models to study the competitive diffusion of desktop and notebook shipments in Taiwan and found evidence of predator-prey relationships [26].

Both models are built based on the data of adoption/shipment amounts and/or product revenues, which may be affected by industry long-term trends, economic cycles, and other confounding factors. Unfortunately, this sort of interference is one of the most important characteristics of DRAM industry and both models may yield inaccurate predictions under this sort of interference. It is therefore questionable that the applicability of revenue data and the effect of data characteristics are rarely analyzed in DRAM industry.

2.4. Models for Multiple Competing Technologies. Under rapid substitution, multiple generations of DRAM processing technologies coexist in the market. Several models are proposed to encompass the diffusion and substitution of multiple competing technologies. Peterka questioned the applicability of revenue data and adopted market share data [7]. Competing technologies are considered pairwise to follow Fisher and Pry's empirical formula, where the logarithmic function of market share ratio is a linear function in time $t$ [8]. The Peterka model can be described by the following equations.

$$
\begin{aligned}
\ln \frac{f_{i}(t)}{f_{j}(t)} & =k_{i j}-c_{i j}\left(t-t_{0}\right), \quad \text { for any } i \text { and } j, \\
\sum_{i} f_{i}(t) & =1,
\end{aligned}
$$

where $t_{0}$ is the starting time of the model and $f_{i}(t)$ is the market share of the $i$ th competing technologies in time $t$. Note that Peterka assumed that no technology can start from zero in this model [4]. A new technology must be initiated and 
established by external investment before $t_{0}$. Thus, $f_{i}\left(t_{0}\right)$ is predetermined according to the external investment and $k_{i j}=$ $\ln \left(f_{i}\left(t_{0}\right) / f_{j}\left(t_{0}\right)\right)$ is the given ratio of initial market shares. As a result, this model may not be suitable for successive generations of DRAM processing technologies, where future generation may be zero at the starting time of the model.

Norton and Bass generalized the Bass model and modeled the demand growth/decline for successive generations of memory and logic circuits [9]. They assume that the number of successive generations is preset and each successive generation has its own market potential and market penetration. A new generation of a product or technology always attracts users from its potential market and the predecessor generation. The Norton-Bass model can be illustrated by a product of three successive generations.

$$
\begin{aligned}
& S_{1}(t)=F_{1}(t) m_{1}\left[1-F_{2}\left(t-\tau_{2}\right)\right], \\
& S_{2}(t)=F_{2}\left(t-\tau_{2}\right)\left[m_{2}+F_{1}(t) m_{1}\right]\left[1-F_{3}\left(t-\tau_{3}\right)\right], \\
& S_{3}(t)=F_{3}\left(t-\tau_{2}\right)\left\{m_{3}+F_{2}\left(t-\tau_{2}\right)\left[m_{2}+F_{1}(t) m_{1}\right]\right\},
\end{aligned}
$$

where the $i$ th generation $i$ is introduced at $\tau_{i}$ and $\tau_{1}=$ 0 . Moreover, $m_{i}$ is the potential sales of the $i$ th generation and $S_{i}(t)$ is the sales of the $i$ th generation in time period $t$. Note that $F_{i}(t)$ is the cumulative distribution function of the adoption rate of the $i$ th generation.

The formula is

$$
F_{i}(t)= \begin{cases}\frac{1-\exp \left[-\left(p_{i}+q_{i}\right) t\right]}{1+\left(p_{i} / q_{i}\right) \exp \left[-\left(p_{i}+q_{i}\right) t\right]}, & \text { for } t \geq \tau_{i} \\ 0, & \text { otherwise, }\end{cases}
$$

where the parameter $p_{i}$ is the coefficient of innovation and $q_{i}$ is the coefficient of imitation of the $i$ th generation.

In these models, there are three common issues. First, the number of technologies must be preset. Each technology is assumed to be predictable and all newborn technologies can go through the complete adoption cycle and competes with earlier ones. Unfortunately, we observe that some new technologies may come to a premature end without adoption in DRAM industry. As a result, the total number of new technologies may not be predetermined.

Second, the inconsecutive adoption of new technologies is not allowed. When adopting the new generation of DRAM processing technologies, customers may intend to bypass the immediate successor and adopt the later one due to the consideration of market potential and transcendence. As a result, some generations of DRAM processing technologies may not go through the complete adoption cycle and compete with earlier ones.

Third, the number of fitting parameters grows quickly with the number of technologies and more data is required for prediction accuracy. For multiple competing technologies, the existing forecasting models are designed to mimic the diffusion and substitution by equations among all technologies. Thus, the number of fitting parameters grows quickly with the number of technologies. However, it is natural that mature technologies have plenty of data and new technologies

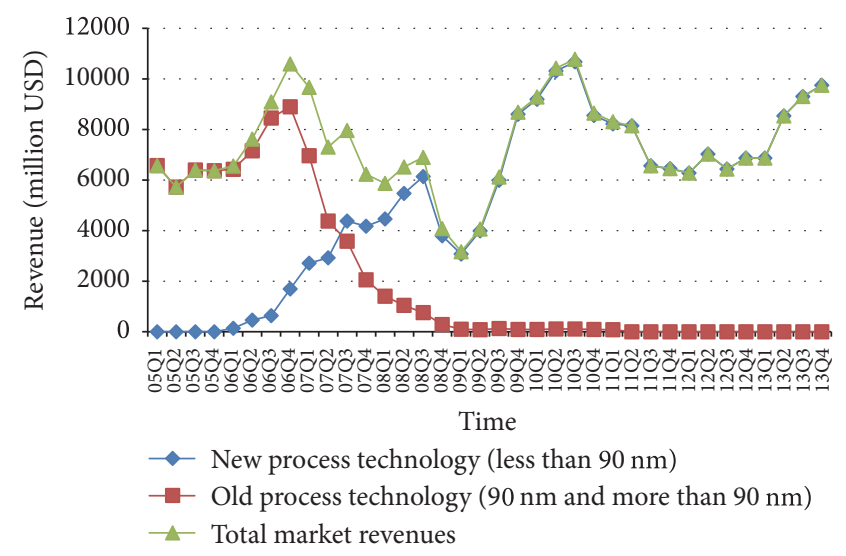

FIGURE 2: DRAM revenue changes for new and old process technologies. Data sources: DRAMeXchange, IHS iSuppli, and Morgan Stanley Research (2014). Data collation: Industrial Technology Research Institute of Taiwan and Taiwan Institute of Economic Research.

have little data collectable in initial stages. As a result, mature technologies dominate the model and errors are cumulated to new technologies. In the worst case, some models may not be applied due to the insufficient data for parameters fitting.

\section{Model}

We now propose the modified Lotka-Volterra model for forecasting the diffusion and substitution of DRAM processing technologies.

3.1. Market Share Data. In this study, we focus on the substitution between old and new DRAM processing technologies. At a given point in time, there usually coexist different generations of processing technology on the DRAM market. For example, 60-, 70-, 80-, 90-, 100-120-, and 130-140nanometer processing technologies coexisted on the DRAM market in 2007 Q3 (see Figure 1). We first divide DRAM processing technologies into two groups, old and new. 90 nanometers are used as the dividing line between the two. Hence, DRAM processing technologies of less than 90 nanometers are classified as new technologies, and those of 90 nanometers and more than 90 nanometers are classified as old technologies.

Data on shipments and/or product revenues are typically used to forecast technological substitution. However, these data may be affected by market fluctuations that have nothing to do with substitution. Such factors include industry longterm trends, economic cycles, and other confounding factors. To illustrate, Figure 2 shows quarterly DRAM revenue data from 2005 Q1 to 2013 Q4. Unstable fluctuations in DRAM revenue are apparent.

Market shares measure the percentages of market sales accounted for by competitive products or technologies. Changes in market shares reflect substitution, not longterm trends, economic cycles, and other confounding factors. 


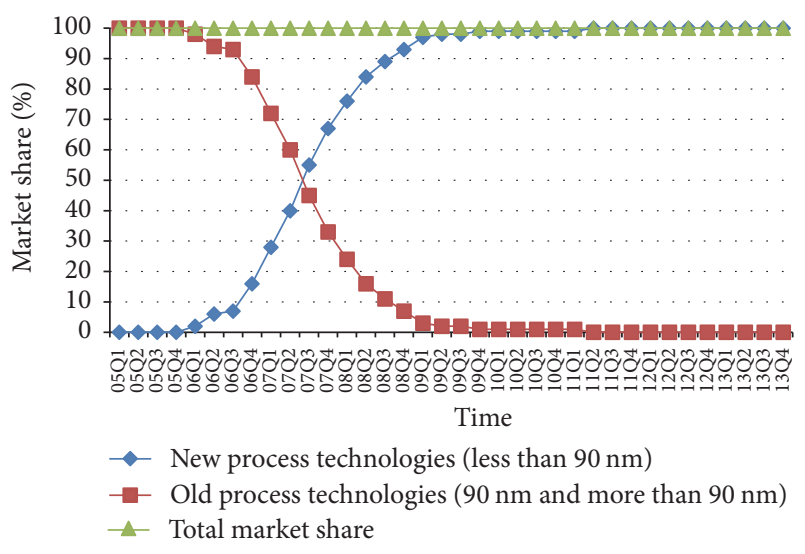

FIGURE 3: DRAM market share changes for new and old process technologies. Data sources: DRAMeXchange, IHS iSuppli, and Morgan Stanley Research (2014). Data collation: Industrial Technology Research Institute of Taiwan and Taiwan Institute of Economic Research.

Figure 3 shows that market shares fluctuate less than does revenue. Hence, our model is to use market share data for forecasting.

3.2. Model Formulation. Using market share data, one natural restriction is that market shares must sum to $100 \%$. By setting $M=1$ and $\bar{y}(t)$ as the cumulative market share of new processing technologies at time $t$, the Bass model given by (1) can be rewritten as follows:

$$
\begin{aligned}
\frac{d \bar{y}(t)}{d t} & =(p+q \bar{y}(t))(1-\bar{y}(t)) \\
& =C_{1}+B_{1} \bar{y}(t)+A_{1} \bar{y}(t)^{2},
\end{aligned}
$$

where $C_{1}=p, B_{1}=q-p$, and $A_{1}=-q$.

When applying market share data to the Lotka-Volterra model, we let $\bar{x}(t)$ and $\bar{y}(t)$ be the market shares of old and new DRAM processing technologies at time $t$, respectively. Given a total market share of $100 \%$, we have

$$
\bar{x}(t)+\bar{y}(t)=1 \text {. }
$$

From (8), (2) can be rewritten as

$$
\begin{aligned}
\frac{d \bar{x}(t)}{d t} & =\left(a_{1}+b_{1} \bar{x}(t)+c_{1} \bar{y}(t)\right) \bar{x}(t) \\
& =\left(\left(a_{1}+c_{1}\right)+\left(b_{1}-c_{1}\right) \bar{x}(t)\right) \bar{x}(t) \\
& =B_{2} \bar{x}(t)+A_{2} \bar{x}(t)^{2},
\end{aligned}
$$

where $B_{2}=a_{1}+c_{1}$ and $A_{2}=b_{1}-c_{1}$.

Equations (7) and (9) show that the Bass and Lotka-Volterra models of market shares degenerate to our model because total market shares must be $100 \%$. This modified Lotka-Volterra model is formulated as follows:

$$
\begin{aligned}
\frac{d \bar{x}(t)}{d t} & =C+B \bar{x}(t)+A \bar{x}(t)^{2} \\
\bar{y}(t) & =1-\bar{x}(t) .
\end{aligned}
$$

3.3. Prediction Evaluation. Traditionally, both the mean absolute percentage error (MAPE) and the mean squared error (MSE) are used to evaluate prediction accuracy. However, depending on the data and models used, the MAPE and MSE are not necessarily the best measures of prediction accuracy. Therefore, we use the MAE to evaluate the accuracy of our market share predictions.

Given a number of forecast periods of $n$ and letting $y(t)$ and $\widehat{y}(t)$ denote the actual and forecast values for time $t$, respectively, the MAPE, MSE, and MAE are defined as

$$
\begin{aligned}
\text { MAPE } & =\frac{1}{n} \sum_{t=1}^{n}\left|\frac{y(t)-\widehat{y}(t)}{y(t)}\right|, \\
\mathrm{MSE} & =\sum_{t=1}^{n} \frac{(y(t)-\widehat{y}(t))^{2}}{n}, \\
\mathrm{MAE} & =\frac{1}{n} \sum_{t=1}^{n}|(y(t)-\hat{y}(t))| .
\end{aligned}
$$

The MAPE conveys the percentage difference between the actual values and forecast values. Small $y(t)$ values can generate large MAPE values. Actual values close to zero can generate infinitely large MAPEs. Therefore, the MAPE is not suitable for prediction evaluation when actual values are small. Given that market shares are, by definition, between 0 and 1 , the MAPE is not suited to our purposes.

The MSE is the mean squared difference between the actual and forecast values. Although it is not troubled by small actual values, because the MSE is dependent on the units of measurement of $y(t)$, it cannot be used for comparisons based on different types of data. Thus, the MSE is not suitable for our study, because we want to compare the prediction accuracy of both revenue and market share forecasts.

The MAE conveys the absolute difference between the actual and forecast values. It is untroubled by small actual values. Moreover, when applied to market share data, the MAE records the average absolute error as a percentage. Thus, to ensure the comparability of our prediction evaluations, we compare the MAEs for market share predictions with the MAPEs for revenue predictions.

\section{Data Collection and Empirical Study}

In this section, our model is implemented to forecast the diffusion and substitution of DRAM processing technologies. Other benchmark forecasting models are used for comparison. These benchmark forecasting models include the standard Bass and Lotka-Volterra models.

Data on revenue and market share have different characteristics and influences. We collect global revenue and market share data on different DRAM processing technologies and use them to evaluate prediction accuracy. Our data sources include DRAMeXchange, iSuppli, and the industry analysis reports published by Taiwan's ITRI. In total, 36 pairs of records from 2005 Q1 to 2013 Q4 were collected.

4.1. Numerical Study. To distinguish between old and new processing technologies, we use three dividing lines: 90, 80, 
TABLE 1: Revenues and market share for old and new processing technologies from 2005 Q1 to 2013 Q4.

\begin{tabular}{|c|c|c|c|c|c|c|c|c|c|c|c|c|}
\hline \multirow{3}{*}{ Quarter } & \multicolumn{6}{|c|}{ Revenue (millions USD) } & \multicolumn{6}{|c|}{ Market share } \\
\hline & \multicolumn{2}{|c|}{$90 \mathrm{~nm}$} & \multicolumn{2}{|c|}{$80 \mathrm{~nm}$} & \multicolumn{2}{|c|}{$70 \mathrm{~nm}$} & \multicolumn{2}{|c|}{$90 \mathrm{~nm}$} & \multicolumn{2}{|c|}{$80 \mathrm{~nm}$} & \multicolumn{2}{|c|}{$70 \mathrm{~nm}$} \\
\hline & Old & New & Old & New & Old & New & Old & New & Old & New & Old & New \\
\hline 05Q1 & 6574 & $<1$ & 6574 & $<1$ & 6574 & $<1$ & 1 & $<0.01$ & 1 & $<0.01$ & 1 & $<0.01$ \\
\hline 05Q2 & 5715 & $<1$ & 5715 & $<1$ & 5715 & $<1$ & 1 & $<0.01$ & 1 & $<0.01$ & 1 & $<0.01$ \\
\hline 05Q3 & 6394 & $<1$ & 6394 & $<1$ & 6394 & $<1$ & 1 & $<0.01$ & 1 & $<0.01$ & 1 & $<0.01$ \\
\hline 05Q4 & 6372 & $<1$ & 6372 & $<1$ & 6372 & $<1$ & 1 & $<0.01$ & 1 & $<0.01$ & 1 & $<0.01$ \\
\hline 06Q1 & 6422 & 131 & 6553 & $<1$ & 6553 & $<1$ & 0.98 & 0.02 & 1 & $<0.01$ & 1 & $<0.01$ \\
\hline 06Q2 & 7158 & 457 & 7615 & $<1$ & 7615 & $<1$ & 0.94 & 0.06 & 1 & $<0.01$ & 1 & $<0.01$ \\
\hline 06Q3 & 8455 & 636 & 9091 & $<1$ & 9091 & $<1$ & 0.93 & 0.07 & 1 & $<0.01$ & 1 & $<0.01$ \\
\hline 06Q4 & 8897 & 1695 & 10592 & $<1$ & 10592 & $<1$ & 0.84 & 0.16 & 1 & $<0.01$ & 1 & $<0.01$ \\
\hline 07Q1 & 6961 & 2707 & 9475 & 193 & 9668 & $<1$ & 0.72 & 0.28 & 0.98 & 0.02 & 1 & $<0.01$ \\
\hline 07Q2 & 4380 & 2920 & 6716 & 584 & 7154 & 146 & 0.6 & 0.4 & 0.92 & 0.08 & 0.98 & 0.02 \\
\hline 07Q3 & 3584 & 4380 & 6929 & 1035 & 7645 & 319 & 0.45 & 0.55 & 0.87 & 0.13 & 0.96 & 0.04 \\
\hline 07Q4 & 2055 & 4173 & 4484 & 1744 & 5294 & 934 & 0.33 & 0.67 & 0.72 & 0.28 & 0.85 & 0.15 \\
\hline 08Q1 & 1408 & 4459 & 3462 & 2405 & 4457 & 1408 & 0.24 & 0.76 & 0.59 & 0.41 & 0.76 & 0.24 \\
\hline 08Q2 & 1042 & 5470 & 2800 & 3712 & 4298 & 2214 & 0.16 & 0.84 & 0.43 & 0.57 & 0.66 & 0.34 \\
\hline 08Q3 & 758 & 6134 & 2205 & 4687 & 3791 & 3101 & 0.11 & 0.89 & 0.32 & 0.68 & 0.55 & 0.45 \\
\hline 08Q4 & 286 & 3793 & 816 & 3263 & 1713 & 2366 & 0.07 & 0.93 & 0.2 & 0.8 & 0.42 & 0.58 \\
\hline 09Q1 & 95 & 3073 & 222 & 2946 & 919 & 2249 & 0.03 & 0.97 & 0.07 & 0.93 & 0.29 & 0.71 \\
\hline 09Q2 & 81 & 3984 & 203 & 3862 & 772 & 3293 & 0.02 & 0.98 & 0.05 & 0.95 & 0.19 & 0.81 \\
\hline 09Q3 & 122 & 5991 & 245 & 5868 & 856 & 5257 & 0.02 & 0.98 & 0.04 & 0.96 & 0.14 & 0.86 \\
\hline 09Q4 & 87 & 8595 & 174 & 8508 & 955 & 7721 & 0.01 & 0.99 & 0.02 & 0.98 & 0.11 & 0.89 \\
\hline 10Q1 & 93 & 9195 & 186 & 9102 & 836 & 8452 & 0.01 & 0.99 & 0.02 & 0.98 & 0.09 & 0.91 \\
\hline 10Q2 & 104 & 10317 & 208 & 10213 & 834 & 9587 & 0.01 & 0.99 & 0.02 & 0.98 & 0.08 & 0.92 \\
\hline 10Q3 & 108 & 10671 & 216 & 10563 & 647 & 10132 & 0.01 & 0.99 & 0.02 & 0.98 & 0.06 & 0.94 \\
\hline 10Q4 & 86 & 8558 & 173 & 8471 & 346 & 8298 & 0.01 & 0.99 & 0.02 & 0.98 & 0.04 & 0.96 \\
\hline 11Q1 & 83 & 8220 & 166 & 8137 & 332 & 7971 & 0.01 & 0.99 & 0.02 & 0.98 & 0.04 & 0.96 \\
\hline 11Q2 & $<1$ & 8147 & $<1$ & 8147 & 163 & 7984 & $<0.01$ & 1 & $<0.01$ & 1 & 0.02 & 0.98 \\
\hline 11Q3 & $<1$ & 6565 & $<1$ & 6565 & 66 & 6499 & $<0.01$ & 1 & $<0.01$ & 1 & 0.01 & 0.99 \\
\hline 11Q4 & $<1$ & 6453 & $<1$ & 6453 & $<1$ & 6453 & $<0.01$ & 1 & $<0.01$ & 1 & $<0.01$ & 1 \\
\hline 12Q1 & $<1$ & 6271 & $<1$ & 6271 & $<1$ & 6271 & $<0.01$ & 1 & $<0.01$ & 1 & $<0.01$ & 1 \\
\hline 12Q2 & $<1$ & 7023 & $<1$ & 7023 & $<1$ & 7023 & $<0.01$ & 1 & $<0.01$ & 1 & $<0.01$ & 1 \\
\hline 12Q3 & $<1$ & 6429 & $<1$ & 6429 & $<1$ & 6429 & $<0.01$ & 1 & $<0.01$ & 1 & $<0.01$ & 1 \\
\hline $12 \mathrm{Q} 4$ & $<1$ & 6864 & $<1$ & 6864 & $<1$ & 6864 & $<0.01$ & 1 & $<0.01$ & 1 & $<0.01$ & 1 \\
\hline 13Q1 & $<1$ & 6870 & $<1$ & 6870 & $<1$ & 6870 & $<0.01$ & 1 & $<0.01$ & 1 & $<0.01$ & 1 \\
\hline 13Q2 & $<1$ & 8531 & $<1$ & 8531 & $<1$ & 8531 & $<0.01$ & 1 & $<0.01$ & 1 & $<0.01$ & 1 \\
\hline 13Q3 & $<1$ & 9300 & $<1$ & 9300 & $<1$ & 9300 & $<0.01$ & 1 & $<0.01$ & 1 & $<0.01$ & 1 \\
\hline 13Q4 & $<1$ & 9748 & $<1$ & 9748 & $<1$ & 9748 & $<0.01$ & 1 & $<0.01$ & 1 & $<0.01$ & 1 \\
\hline
\end{tabular}

Data sources: DRAMeXchange, IHS iSuppli, \& Morgan Stanley Research (2014).

Data collation: Industrial Technology Research Institute of Taiwan \& Taiwan Institute of Economic Research.

and 70 nanometers as examples. The raw data for each are given in Table 1.

We are also interested in prediction accuracy at different stages of diffusion. The $99 \%, 95 \%, 90 \%, 80 \%, 70 \%, 60 \%$, and $50 \%$ market shares for new processing technologies are earmarked as seven stages of diffusion. In each stage, records pertaining to the time before the new processing technologies surpassed the earmarked market shares were used as training data to fit the model parameters. The remaining records were used as testing data to evaluate prediction accuracy.
For example, at the dividing line of 90 nanometers, new processing technologies are those using 90 nanometers or less. Table 1 shows that these new processing technologies reached a 99\% market share in 2009 Q4. Thus, in Stage 1, 19 pairs of revenue records for the old and new processing technologies from 2005 Q1 to 2009 Q3 were used as training data to fit the Bass and Lotka-Volterra models by using ordinary least squares. The remaining 17 pairs of revenue records were used as testing data to individually evaluate the MAPEs for the old and new processing technologies. 
Similarly, 19 market share records from 2005 Q1 to 2009 Q3 were used as training data to fit the Bass, Lotka-Volterra, and modified Lotka-Volterra models by using ordinary least squares in Stage 1. The remaining 17 market share records were used as testing data to individually evaluate the MAEs for the old and new processing technologies. The results for all seven stages are reported in Table 2. Tables 3 and 4 report the results of our numerical studies for the 80 - and 70-nanometer dividing lines, respectively.

4.2. Analysis of Data Selection. The results reported in the previous subsection indicate that the lower the number of records used as training data, the higher the prediction errors. Moreover, the market share predictions are significantly better than the revenue predictions from both the Bass and Lotka-Volterra models. Consider the 90-nanometer dividing line as an example (see Table 2). When using revenue data on the Bass model, the prediction errors range from $9.07 \%$ to infinity for the seven stages. When using market share data on the Bass model, the prediction errors are much smaller: between $0.56 \%$ and $17.11 \%$ for the seven stages. Similarly, for the Lotka-Volterra model, using revenue data generates prediction errors of from $9.52 \%$ to infinity, whereas using market share data gives prediction errors of between $0.14 \%$ and $91.90 \%$. Results based on the other diving lines are similar (see Tables 3 and 4).

Arguably, revenue data are not suitable for forecasting the diffusion and substitution of technologies. This is because revenue data reflect market fluctuations caused by factors other than substitution, including industry long-term trends, economic cycles, and other confounding factors.

For example, global DRAM industry revenue has exhibited a prolonged growth trend, particularly in terms of demand for computers and mobile communications products. In 2007, the DRAM industry invested huge amounts of capital to expand production capacity for upgrading to a new PC operating system, Windows Vista. However, sales of Windows Vista fell far short of market expectations. This led to an oversupply of DRAM products, which caused spot prices and revenue to crash in 2007. In 2008, global DRAM revenue continued to decline because of the global financial tsunami. The consequent unexpected fall in global DRAM revenue from 2006 Q4 to 2009 Q1 greatly affected prediction accuracy.

Market share data are more suitable than revenue data for forecasting the diffusion and substitution of DRAM processing technologies. This is because the market share data record percentages and because the total market share is fixed at $100 \%$. Because market share data are unaffected by seasonal factors and economic cycles, they reflect mainly technological substitution. Consequently, using market share data generates more accurate predictions and is appropriate for analyzing technological substitution.

4.3. Analysis of Model Selection. Having shown that using market share data increases prediction accuracy for all forecasting models, in this subsection, we use market share data to compare the Bass, Lotka-Volterra, and modified Lotka-Volterra models.
First, note the symmetry of the modified Lotka-Volterra model based on market share data. In each stage, the prediction errors for the old and new processing technologies are the same. This is because the market shares are forced to sum to $100 \%$ in (10) of the modified Lotka-Volterra model. As a result, the forecast values for the old and new processing technologies sum to $100 \%$ and must have the same MAEs. Similarly, for the Bass model based on market share data, the prediction errors for the old and new processing technologies are highly symmetrical. Again, this arises because of the characteristics of the market share data.

By contrast, the Lotka-Volterra model based on market share data does not generate symmetric prediction errors for the old and new processing technologies. This is because the market shares of old and new processing technologies are treated as two species and there is no total population limit for the two species in the Lotka-Volterra model. The forecast market shares are not constrained to sum to $100 \%$. This is why the prediction errors from the Lotka-Volterra model are relatively high.

Let us compare the accuracy of the models based on market share data. In Stages 1 to 7 of Table 2 (which reports results for the 90-nanometer dividing line), the Bass model generates prediction errors of between $0.56 \%$ and $17.11 \%$, the Lotka-Volterra model generates prediction errors of between $0.14 \%$ and $91.90 \%$, and the modified Lotka-Volterra model generates prediction errors of between $0.16 \%$ and $0.50 \%$ (Tables 3 and 4 show that the other dividing lines generate similar results.) Therefore, when forecasting market shares, the modified Lotka-Volterra model outperforms the Bass and Lotka-Volterra models.

\section{Conclusions and Future Research}

In this study, we propose a modified Lotka-Volterra model to forecast the diffusion and substitution of DRAM processing technologies. Multiple generations of DRAM processing technologies are partitioned into old and new generations and the quarterly data from 2005 Q1 to 2013 Q4 is used for empirical studies. In our model, there are four features.

First, the type of data affects the prediction accuracy of the technological substitution trends. Although shipment and/or revenue data have been widely used in existing research, they may be contaminated by confounding factors that reduce prediction accuracy. This can be avoided by using market share data, which are purged of such factors as long-term trends and business cycles. Consequently, using market share data can generate more accurate forecasts of technological substitution.

Second, existing models for multiple competing technologies require predetermined number of technologies and each technology is assumed to go through the complete adoption cycle. However, both are not obvious in DRAM industry. Focusing on these issues, our modified Lotka-Volterra model allows undetermined number of multigeneration technologies and inconsecutive adoption of new technologies. 


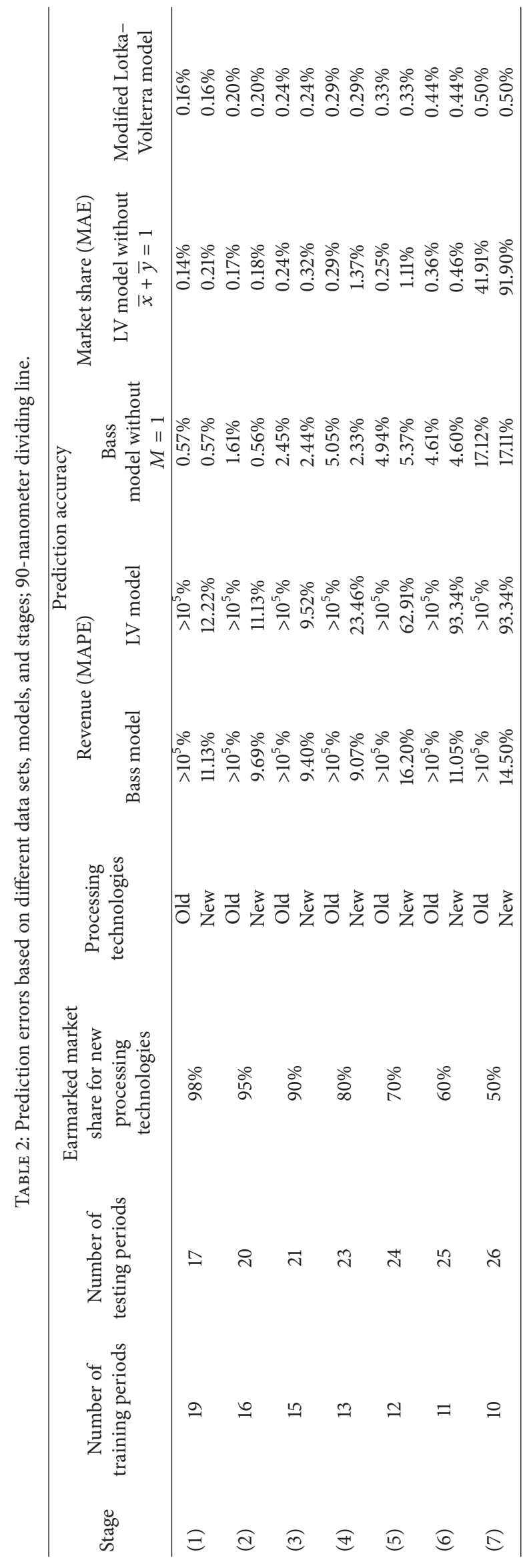


Mathematical Problems in Engineering

9

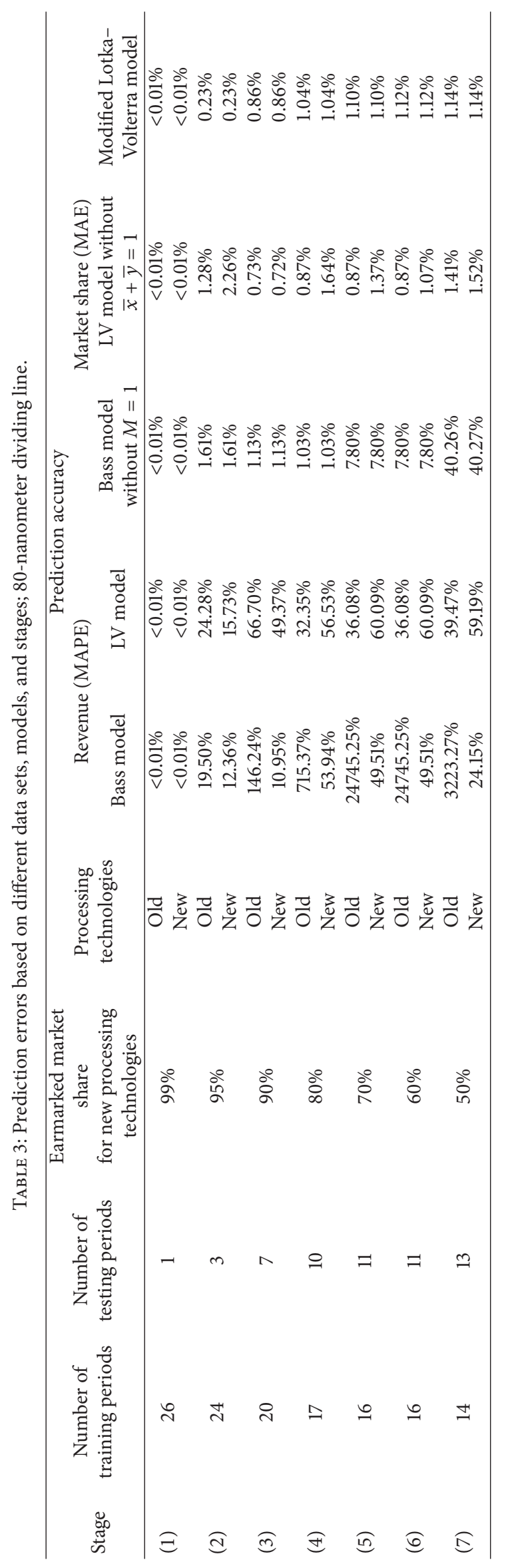




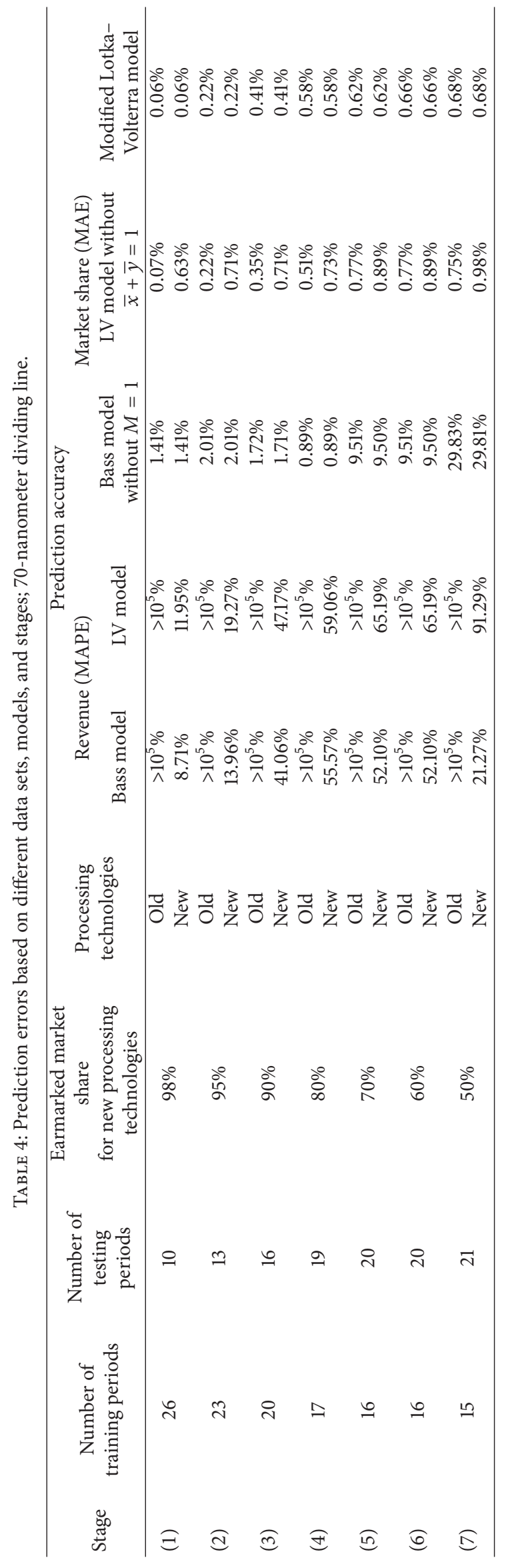


Third, the number of fitting parameters in our model is fixed and relatively small. This increases not only the prediction accuracy, but also the applicability of our model with little data. Moreover, the forecasting of a single generation of technology can be computed by the difference between two our models with consecutive dividing lines.

Fourth, MAE is suitable for evaluating the prediction accuracy of different forecasting models based on market share data. There are limitations to adopt MAPE and MSE for evaluating prediction accuracy. The MAPE may generate misleading measures of prediction accuracy for small actual values, and the MSE is not suitable for evaluating forecasting models that use different types of data. Therefore, we used the MAE to evaluate the accuracy of our market share predictions.

In the future, we intend to apply our model to other industries and confirm our findings related to forecasting performance and prediction accuracy. This is important because different industries have different characteristics. We are interested in studying these characteristics and modifying our model to fit individual industries. For example, integrated device manufacturers and IC industries are highly capital intensive and their demands are highly influenced by seasonal factors and business cycles. Another example is the display industry, in which old and new technologies are in fierce competition. However, alliances also exist among enterprises for technology support. These interesting and special characteristics can be expected to reduce signal-to-noise ratios and to increase prediction accuracy. We aim to investigate these issues and develop new procedures for improving prediction accuracy further.

\section{Conflicts of Interest}

The authors declare that they have no conflicts of interest.

\section{References}

[1] N. M. Victor and J. H. Ausubel, "DRAMs as model organisms for study of technological evolution," Technological Forecasting and Social Change, vol. 69, no. 3, pp. 243-262, 2002.

[2] A. Grant, "Memory upgrade: the demands of modern computing call for a seismic shift in data storage and retrieval," Science News, vol. 184, pp. 28-31, 2013.

[3] TrendForce, "TrendForce Reports mobile DRAM industry valued over US\$3.6 billion in 4Q14 due to strong smartphone shipments," 2015 http://press.trendforce.com/node/create_pdf/ 1842.

[4] IHS iSuppli, "DRAM Revenue in Q1 Down 6 Percent to \$8.3 Billion,” 2011 https://technology.ihs.com/394338/dram-revenuein-q1-down-6-percent-to-83-billion.

[5] DRAMeXchange, "DRAMeXchange: Little Change in 4Q11 DRAM Revenue Ranking, Mainly due to Mobile and Server DRAM Production Increase by First-tier Manufacturers," 2012 http://press.trendforce.com/press/-516.html.

[6] DRAMeXchange, "TrendForce: DRAM Industry Value Grows for Fifth Consecutive Quarter in 4Q13," 2014 http://www.dramexchange.com/WeeklyResearch/Post/2/3655.html.
[7] V. Peterka, "Macrodynamics of Technological Change: Market Penetration by New Technologies, International Institute for Applied Systems Analysis," Laxenburg, Austria, 1977.

[8] J. C. Fisher and R. H. Pry, "A simple substitution model of technological change," Technological Forecasting and Social Change, vol. 3, pp. 75-88, 1971.

[9] J. A. Norton and F. M. Bass, "A diffusion theory model of adoption and substitution for successive generations of hightechnology products," Management Science, vol. 33, pp. 10691086, 1987.

[10] F. M. Bass, "A new product growth model for consumer durables," Management Science, vol. 15, pp. 215-227, 1969.

[11] V. Mahajan, E. Muller, and F. M. Bass, "Diffusion of new products: empirical generalizations and managerial uses," Marketing Science, vol. 14, pp. G79-G88, 1995.

[12] S. Y. Park, J. W. Kim, and D. H. Lee, “Development of a market penetration forecasting model for Hydrogen Fuel Cell Vehicles considering infrastructure and cost reduction effects," Energy Policy, vol. 39, no. 6, pp. 3307-3315, 2011.

[13] A.-C. Cheng, "Exploring the relationship between technology diffusion and new material diffusion: the example of advanced ceramic powders," Technovation, vol. 32, no. 3-4, pp. 163-167, 2012.

[14] B.-H. Tsai, Y. Li, and G.-H. Lee, "Forecasting global adoption of crystal display televisions with modified product diffusion model," Computers \& Industrial Engineering, vol. 58, pp. 553$562,2010$.

[15] D. Y. Kong and X. H. Bi, "Impact of social network and business model on innovation diffusion of electric vehicles in China," Mathematical Problems in Engineering, vol. 2014, Article ID 230765, 7 pages, 2014.

[16] A. J. Lotka, Elements of physical biology, Williams and Wilkins, Baltimore, USA, 1925.

[17] V. Volterra, "Fluctuations in the abundance of a species considered mathematically," Nature, vol. 118, no. 2972, pp. 558-560, 1926.

[18] K. Smitalova and S. Sujan, A Mathematical Treatment of Dynamical Models in Biological Science, Eills Horwood, West Sussex, UK, 1991.

[19] I. R. Geijzendorffer, W. van der Werf, F. J. J. A. Bianchi, and R. P. O. Schulte, "Sustained dynamic transience in a Lotka-Volterra competition model system for grassland species," Ecological Modelling, vol. 222, no. 15, pp. 2817-2824, 2011.

[20] R. M. Goodwin, Socialism, Capitalism and Economic Growth, Cambridge University Press, Cambridge, UK, 1967.

[21] V. B. Kreng and H. T. Wang, "The interaction of the market competition between LCD TV and PDP TV," Computers \& Industrial Engineering, vol. 57, pp. 1210-1217, 2009.

[22] J. Kim, D. J. Lee, and J. Ahn, "A dynamic competition analysis on the Korean mobile phone market using competitive diffusion model," Computers \& Industrial Engineering, vol. 51, pp. 174-182, 2006.

[23] C. Watanabe, R. Kondo, and A. Nagamatsu, "Policy options for the diffusion orbit of competitive innovations-An application of Lotka-Volterra equations to Japan's transition from analog to digital TV broadcasting," Technovation, vol. 23, no. 5, pp. 437445, 2003.

[24] B.-H. Tsai and Y. Li, "Cluster evolution of IC industry from Taiwan to China," Technological Forecasting \& Social Change, vol. 76, pp. 1092-1104, 2009. 
[25] S.-Y. Chiang, "An application of LotkaVolterra model to Taiwans transition from $200 \mathrm{~mm}$ to $300 \mathrm{~mm}$ silicon wafers," Technological Forecasting \& Social Change, vol. 79, pp. 383-392, 2012.

[26] S.-Y. Chiang and G.-G. Wong, "Competitive diffusion of personal computer shipments in Taiwan," Technological Forecasting \& Social Change, vol. 78, pp. 526-535, 2011. 


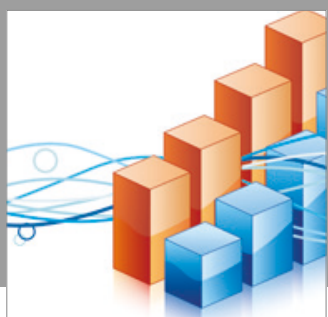

Advances in

Operations Research

vatersals

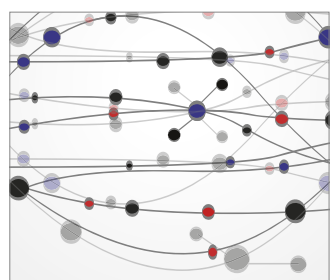

\section{The Scientific} World Journal
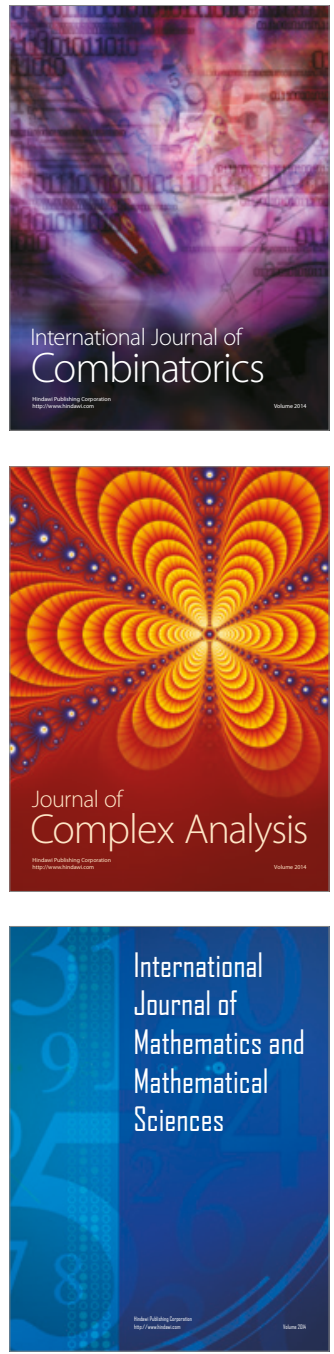
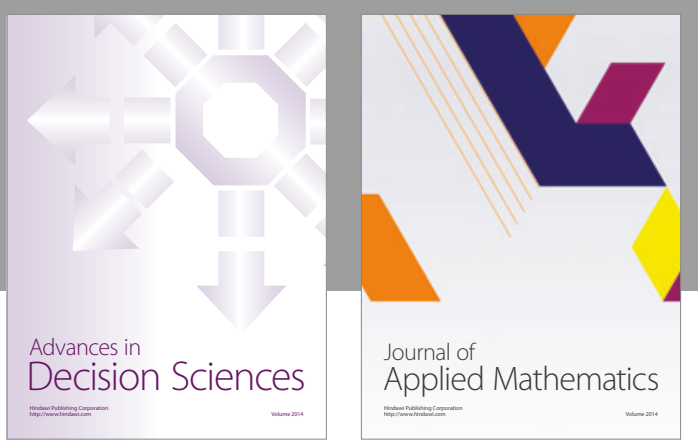

Algebra

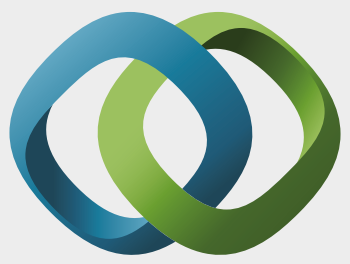

\section{Hindawi}

Submit your manuscripts at

https://www.hindawi.com
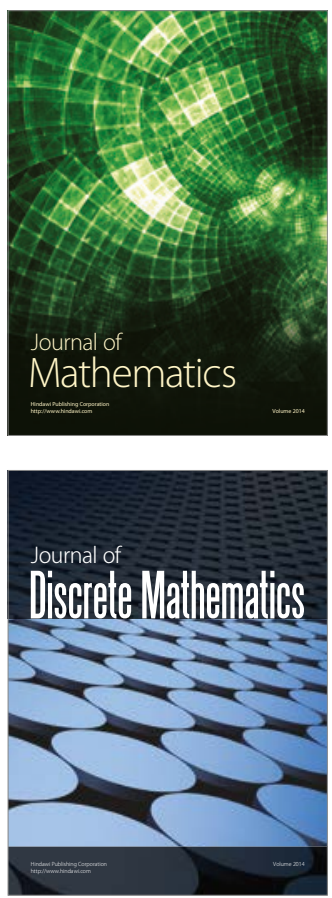

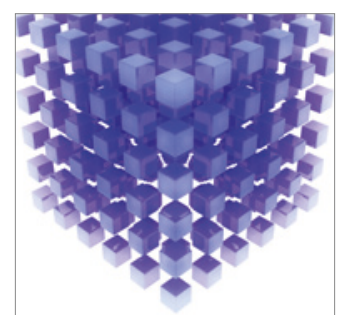

Mathematical Problems in Engineering
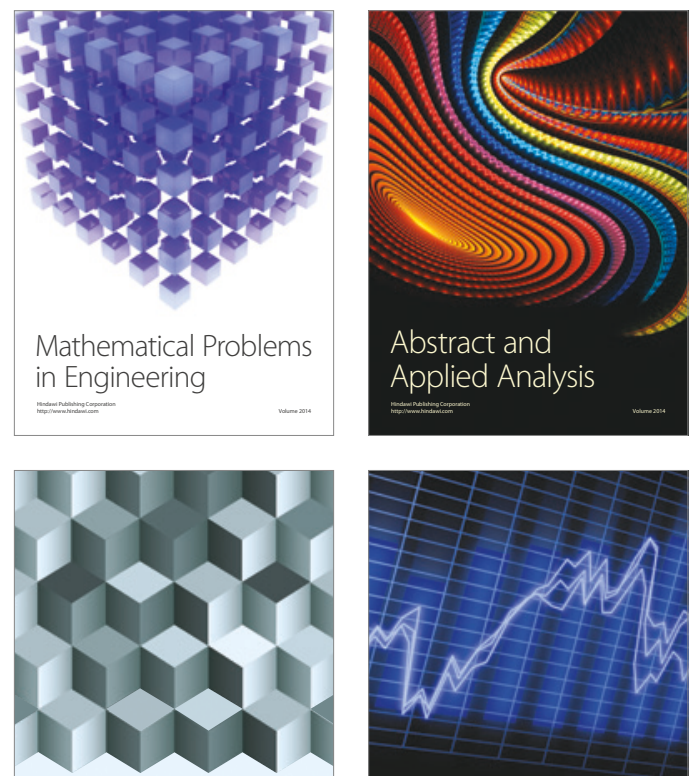

Journal of

Function Spaces

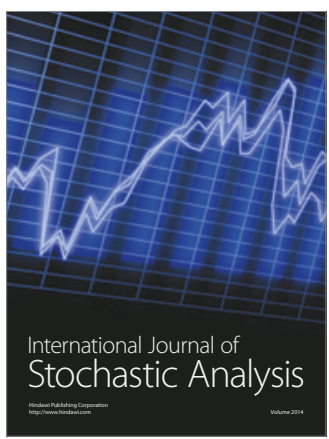

Probability and Statistics
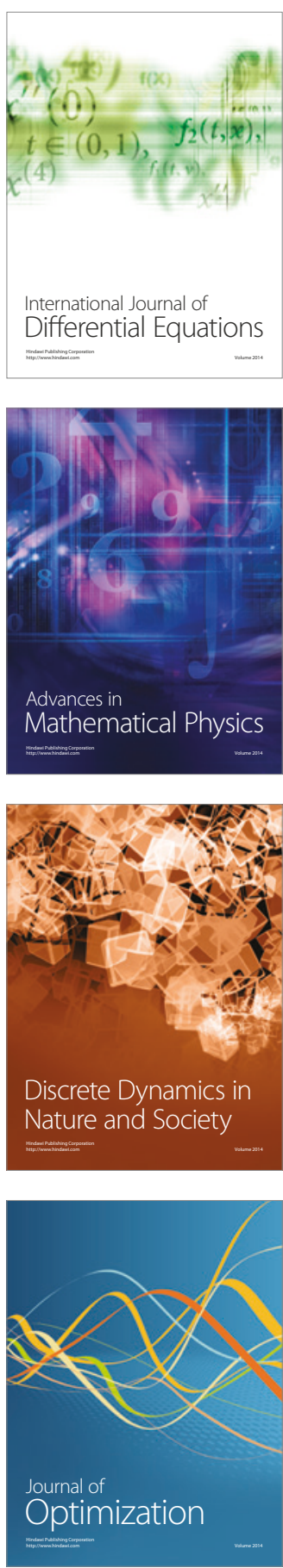